\title{
Impact and storage stability of double fortified salt
}

\begin{abstract}
Anemia is a public health problem that can affects at any stage of human life span. Children, adolscents and pregnant women are the most vulnerable group suffering from iron deficiency anemia. Anemia exists in the society due to low hemoglobin level, inadequate intake of iron rich food, lack of awareness regarding healthy eating practices and due to poverty also. For this strategy, salt is one of the food vehicles available for food fortification technique. Double fortified salt enriched with iron and iodine is stable under various conditions and thus proven a healthy approach to reduce the prevalence of Anemia at a very low cost among the vulnerable population around the world.
\end{abstract}

Keywords: anemia, double fortified salt, iodine, iron, storage, stability, supplementation
Volume 8 Issue 3 - 2018

\author{
Shikha Bathla \\ Assistant Professor, Punjab Agricultural University, India
}

Correspondence: Shikha Bathla, Assistant Professor, Punjab Agricultural University and \#Kiran GroverI 141004, India, Email Shikha_bathla@yahoo.com

Received: February 17, 2017| Published: June 22, 2018

\section{Introduction}

Globally, Anemia affects 1.62 billion people that correspond to 24.8 per cent of the population. The highest prevalence is in preschool age children-293 million (47.4\%), among school children prevalence affected- 305 million $(25.4 \%)$ and with the greatest number of individuals affected is non-pregnant women- 468.4 million (30.2\%). Iron deficiency is a principal cause of Anemia. ${ }^{1}$ Iron deficiency continues to be the leading single nutritional deficiency in the world, despite considerable efforts over the past three decades has been done to decrease its prevalence. National programmes to control and prevent anemia have not been successful after a period of even three decades due to several factors responsible for it like use of low fortification level, low consumption of iron fortified food by the population due to lack of access and its availability, socio-economic factors, and parasitic infections etc. So, Ministry of women and children has now recommended combination of different approaches including dietary diversification, fortification and supplementation. Supplementation of double fortified salt in daily meal preparations helps in the reduction of anemia among the vulnerable group like adolescents and school children. ${ }^{2-4}$ For this supplementation strategy, salt is one of the food vehicles available for food fortification technique. Salt dual-fortified with iodine and micronized ground ferric pyrophosphate (FePP) is sound approach to control the micronutrient deficiency along with improving upon nutritional status. The Micronutrient Initiative formulation uses physical separation of iodine by microencapsulation technique and this salt is stable for usage for a period of six months in different storage containers like metal, plastic, glass and zip-lock pouches for retention of micro-nutrients..$^{5-8}$ So the present work has been an attempt to review the stability and impact of double fortified salt in reducing the prevalence of Anemia.

\section{Double fortified salt storage stability}

Double fortified salt was prepared with various formulations of iodine and microencapsulated iron had been investigated for stability in a period of five-month, under actual field conditions of storage and distribution, in the south coastal and north highland regions of Nigeria. A control of iodated salt and fifteen 500g sample packets of double fortified salt, prepared in duplicate, were sealed in polyethylene bags. These samples and a data logger for monitoring the important parameters like temperature and humidity were packaged into a typical bundle which was overwrapped with polyethylene film and inserted into the distribution network starting from a salt manufacturer's facility to the consumer. Iodine retention values of up to 79 percent were obtained when iodated salt was used and 64 percent when potassium iodide premix was used. It was found that less than 20 percent of the ferrous iron was oxidized to ferric iron, and about 25 per cent of the iron in formulations with sodium ferric EDTA (NaFeEDTA) reduced to ferrous iron. Double fortified salt with ferrous fumarate was generally more stable than those prepared with the other iron premixes. The results concluded that the polyethylene film overwrap of salt packs in the bundles provided a significant protection from ambient humidity. Iodine and the ferrous iron were protected in double fortified salt with iodine and microencapsulated iron. ${ }^{9}$ The fortification technology for double fortified salt was developed and named it as growth plus, that helps in preventing the iron-mediated undesirable taste and appearance of final product after preserving for the stability and bioavailability. The results reported that the iron was stabilized by encapsulation, chelation and redox modulation. This technology had no adverse effect on taste, appearance and product stability and did not interfere with the bioavailability of iron from either ferrous bis-glycinate or ferrous fumarate. Multiple interventions had reported that redox technology helped in stabilizing the iron in the form of a powdered fruit beverage and reduced the incidence of iron deficiency anaemia in school children, adolescent girls and pregnant women..$^{10}$ The stability of table salt and double fortified with various formulations of iodine and microencapsulated iron under actual field conditions of storage and distribution was studied in the coastal and highland regions of Kenya. Twenty-nine samples were taken and each weighed about $200 \mathrm{~g}$ of double fortified salt packed in sealed polyethylene bags. Using a data logger for monitoring the temperature and humidity were packaged into a typical bundle which was inserted into the distribution network starting from a salt manufacturer's facility to the consumer. High iodine retention values were obtained during the three-month study. Most of the iron was retained with less than 20 per cent of the ferrous iron oxidized to ferric iron and 
about 25 percent of the iron in formulations with sodium ferric EDTA (NaFeEDTA) reduced to ferrous iron. It was concluded from the study that double fortified salt with ferrous fumarate was more stable than those prepared with the other iron premixes. The polyethylene film overwrap of salt packs in the bundles provided significant protection from the humidity conditions. Salt double fortified with both iodine and microencapsulated iron ferrous fumarate technique premix was quite stable because both iodine and the ferrous iron were protected during the three months in the distribution and retail network in typical tropical conditions in Kenya's highlands and humid lowlands. ${ }^{11}$ Salt fortified with iron may greatly reduce the prevalence of iron deficiency globally, but fortification is complicated by iron-iodine interactions. To minimize the interaction, a spray dry microencapsulation system was developed. Bio available ferrous fumarate powders were encapsulated via spray-drying to produce particles which are too small to be detected visually. Feed systems containing both suspended and dissolved ferrous fumarate were compared to find optimal feed conditions. The premixes were blended into iodized salt at 1000ppmiron and stored at $40^{\circ} \mathrm{C}$ and $\sim 60$ percent relative humidity. The salt was stored for a period of six months to evaluate the effect on iodine stability. The results concluded that all the encapsulated samples in the conducted experiment were more stable than the unencapsulated samples. ${ }^{12}$

\section{Impact of supplementation of double fortified salt}

The stability of double-fortified salt during storage and its efficacy in improving the iron and iodine status of the communities was studied for duration of two years in seven clusters. However, the bio efficacy of double-fortified salt was assessed in communities covering three states of the country for a period of one year. The experimental group used double-fortified salt and the control group used iodized salt. The salts were used in all meals prepared for family members, but determination of $\mathrm{Hb}$ (haemoglobin) by the cyan methemoglobin method was performed in only two or three members per family, and not in children under 10 years of age $(n=393$ and 436 in the experimental and control groups, respectively). The family size was usually four or five, with a male: female ratio of 1:1, consisting of two parents with two or three children. $\mathrm{Hb}$ (haemoglobin) was measured at a period of including baseline, six and twelve months. Urinary iodine was measured in only one cluster at baseline and endpoint. All the participants were de wormed at baseline, six and twelve months to evaluate the efficacy of double-fortified salt. The results concluded that both iron and iodine in the double-fortified salt were stable during storage for 2 years. Over a period of 1 year, there was an increase of $1.98 \mathrm{~g} / \mathrm{dL}$ of haemoglobin in the experimental group and $0.77 \mathrm{~g} / \mathrm{dL}$ of haemoglobin in the control group; the latter increase may have been due to de worming effect. The median urinary iodine changed from 200 at baseline to $205 \mu \mathrm{g} / \mathrm{dL}$ at the end of the study in the experimental group and from 225 to $220 \mu \mathrm{g} / \mathrm{dL}$ in the control group. There was a significant $(\mathrm{p}<.05)$ improvement in the median urinary iodine status of subjects who were iodine deficient (urinary iodine $<100 \mu \mathrm{g} / \mathrm{L}$ ) in experimental as well as in the control groups. The study concluded that the double-fortified salt was efficient in increasing $(\mathrm{p}<.05)$ the haemoglobin status in all seven clusters in the experimental group when compared with control ones. ${ }^{13}$ The efficacy of NaFeEDTA fortified cowpea meal in improving the iron status in a malaria endemic area was investigated among school going children in the age group of 5-12 years old $(n=241)$ from two rural communities in northern Ghana. Subjects were assigned to consume the cow meal fortified with $10 \mathrm{mgFe} / \mathrm{meal}$ as NaFeEDTA in the experimental group $(n=121)$ and non-fortified cowpea meal to the control group $(n=120)$ provided for 3 day/week for a duration of seven months. For the trial, all the subjects had taken de worming and malaria antigenemia screening. The treatment was carried out at baseline and 3.5 months. At the last stage of trial, out of all the subjects $(n=261)$, experimental group $(\mathrm{n}=115)$ and among control ones $(\mathrm{n}=109)$ have completed the study. In the study, experimental group had shown improvement in haemoglobin level $(\mathrm{P}<0.05)$, serum ferritin level $(\mathrm{P}<0.001)$ and body iron stores $(\mathrm{P}<0.001)$. The trial also showed that the reduction in transferring receptor $(\mathrm{P}<0.001)$ compared with the control group. The study concluded that cow meal fortified with $10 \mathrm{mgFe} / \mathrm{meal}$ as NaFeEDTA helps in improving the iron status and also reduce the prevalence of anaemia among the subjects. ${ }^{14,15}$ developed a technique to fortify wheat flour with absorption promoters of iron, such as ascorbic acid, disodium ethylene diamine tetra acetic acid (NaEDTA) and with a stabilizer, sodium hexametaphosphate (SHMP), with or without iron. In vitro bioavailability of iron was studied in food (Indian bread, chapathi) like wheat flour fortified at $60 \mathrm{mg}$ of iron/ $\mathrm{kg}$ in the presence (1:1 molar ratio) or absence of the three chemical additives was tested. NaEDTA and ascorbic acid enhanced the in vitro bioavailability of native iron from Indian bread while SHMP had no effect. The results showed that the three additives help in enhancing the in vitro bio availability of total iron (native and added iron) from iron fortified chapattis. The predicted bioavailability of iron in man from Indian bread containing ascorbic acid or NaEDTA was twice as high than that with wheat flour alone or that with SHMP $(8 \%)$. Similar enhancing effects of these two compounds were shown with iron-fortified wheat flour. It was concluded from the experiment trial that wheat flour fortified with ascorbic acid or NaEDTA, either with or without iron, can enhance the predicted bioavailability of both native and added iron in man.

The consumption pattern of DFS (Dual fortification of salt) was studied in Moroccan school children in the age group of 6-15years $(\mathrm{n}=377)$. The salt intake was measured by 3 -day weighed food record method and estimated the iron bioavailability from the local diet by using published algorithms. DFS containing $25 \mu \mathrm{g}$ iodine $/ \mathrm{g}$ salt (as potassium iodide) and $1 \mathrm{mg}$ iron/g salt (as ferrous sulphate hydrate encapsulated with partially hydrogenated vegetable oil). After storage and acceptability trials, the efficacy of the DFS was compared in comparison to iodized salt in duration of 9 months. The findings reported that after conducting the trials, the mean salt intake in school-age children was 7-12 g/d, and iron bioavailability from the local diet was $0.4-4.3$ per cent. After storage period for 20 weeks, the DFS and iodized salt were not significantly different in iodine content, and colour stability was acceptable when the compounds were added to local meals. During the efficacy trial, urinary iodine concentrations and thyroid volumes were improved significantly ( $\mathrm{P}<0.001$ and $<0.05$, respectively). At 40 weeks, mean haemoglobin concentrations in the DFS group had increased by $14 \mathrm{~g} / \mathrm{L}(\mathrm{P}<0.01)$, and serum ferritin, transferring receptor, and zinc protoporphyrin concentrations were significantly better $(\mathrm{P}<0.05)$. The prevalence of iron deficiency anemia improves significantly by supplementing the DFS from 35 at baseline to 8 per cent at a period of 40 weeks Zimmermann et al. ${ }^{16}$ One more study among $18-50$ years old women $(n=330)$ to evaluate the efficacy of iron powders that was fortified in cereal products investigated by Zimmermann et al. ${ }^{17}$ Subjects were randomly assigned into four groups to receive either no fortification on or $12 \mathrm{mg} \mathrm{Fe} /$ day for 6 day/week for 35 week as ferrous sulphate, electrolytic iron, or hydrogen-reduced iron in a baked and wheat-flour based snack. 
The snacks were not consumed with meals and consumption was monitored. At baseline, 20 and 35 weeks, haemoglobin and iron status was measured to analyze the difference between groups. The results reported that at baseline and 35 weeks, geometric mean serum ferritin (SF) increased significantly in all 3 groups receiving iron $(\mathrm{P}<0.01)$ and geometric mean serum transferring receptor (TfR) decreased significantly in the groups receiving ferrous sulfate and electrolytic iron $(\mathrm{P}<0.05)$. Calculated mean $( \pm \mathrm{SD})$ body iron stores increased from $1.5 \pm 2.8$ to $5.4 \pm 2.9 \mathrm{mg} / \mathrm{kg}$ in the ferrous sulfate group, from $1.5 \pm 3.5$ to $4.4 \pm 3.6 \mathrm{mg} / \mathrm{kg}$ in the electrolytic iron group, and from $1.3 \pm 3.2$ to $3.2 \pm 4.3 \mathrm{mg} / \mathrm{kg}$ in the hydrogen-reduced iron group $(\mathrm{P}<$ 0.01 for all 3 groups) but did not change significantly in the control group as compared to experimental group. The study concluded that the ferrous sulfate, electrolytic iron, hydrogen-reduced iron and fortification into wheat-based snacks were significantly improved the iron status of the subject. It was also reported that the change in body iron stores during the 35-week study, the relative efficacy of the electrolytic and hydrogen-reduced iron compared with ferrous sulfate was 77 and 49 percent, respectively. Deficiencies of iron and iodine are common in West Africa, and salt was a feasible approach for fortification technique. Salt dual-fortified with iodine and micronized ground ferric pyrophosphate (FePP) was tested for its efficacy in rural, tropical Côte d'Ivoire double-blind trial in the age group of 5 to 15 years old iron-deficient children $(\mathrm{n}=123)$. Salt and iron intakes, and iron bioavailability were estimated using 3-day weighed food records in 24 households. Local iodized salt was fortified with $3 \mathrm{mgFe} / \mathrm{g}$ salt as ground FePP (mean particle size $=2.5 \mu \mathrm{m}$ ), and stability, sensory and acceptability trials were done. The dual fortified salt (DFS) was distributed to households and its efficacy compared with that of iodized salt (IS) in a 6-months. All children were de wormed at baseline. After 6 month, serum ferritin (SF) and transferring receptor (TfR) concentrations as well as body iron stores improved significantly by consuming DFS. Body iron increased from 4.6 \pm 2.7 to $5.9 \pm 2.7 \mathrm{mg} / \mathrm{kg}(\operatorname{mean} \pm \mathrm{SD})$ in the DFS group; concentrations before and after treatment in the IS group were $5.5 \pm 2.9$ and $5.6 \pm 3.1 \mathrm{mg} / \mathrm{kg}$, respectively. The haemoglobin concentration and the prevalence of anaemia did not change in either group. In tropical West Africa, lowgrade salt fortified with micronized ground FePP increased body iron stores but not haemoglobin among the subjects. ${ }^{5}$ Ferrous fumurate and ferrous succinate caused few or no changes in the stored cereal products or made porridge by adding milk or hot water during cooking as compared to ferrous sulphate while fortify foods for infants and young children. In the human trials, iron absorption from a cereal fortified with $7.5 \mathrm{mg}$ iron as ferrous sulphate and $35 \mathrm{mg}$ ascorbic acid per $100 \mathrm{~g}$ dry cereal was 3 percent. The relative bioavailability values were 100 for ferrous fumurate and 92 for ferrous succinate as compared to 39 for ferric pyrophosphate and 75 percent for ferric saccharate. Ascorbic acid was also added along with all iron compounds. All the tested compounds showed no sensory problems but ferrous sulphate did cause change in the colour among cereal products or made porridge by adding milk or hot water during cooking. The results concluded that ferrous fumurate was recommended to fortify foods for infants and young children. ${ }^{18}$ Iodine was coated with dextran such that after spraying onto table salt iron and iodine do not interact with each other. For the trial $(\mathrm{n}=16)$ subjects were given Fe-enhancing and Feinhibiting meals containing $5 \mathrm{~g}$ of table salt with o.39umol dextran coated iodine as potassium iodide and $1 \mathrm{mg}$ of $\mathrm{Fe}$ per gram of salt The subjects were also given a reference dose of $3 \mathrm{mg}$ of ferrous fumurate labeled with $\mathrm{Fe}$ to correct the intra individual variation in iron absorption afterwards. The results reported that $\mathrm{Fe}$ absorption from the Fe-enhancing meal was $(36.2 \pm 12.0$ corrected, $13.5 \pm 13.8 \%$ uncorrected) and Fe-inhibiting meals (7.4 \pm 11.3 corrected, $4.0 \pm 8.4 \%$ uncorrected) $(\mathrm{P}<0.0001)$. It was also observed that urinary excretion of iodine baseline and after intervention was not significantly different $(0.85 \pm 0.5$ to $1.06 \pm 0.39 \mathrm{umol} / \mathrm{L})$. The results reported that Fe was well absorbed after intervention trial but urinary iodine was maintained in normal range with dextran coated meal.

The fortified salt helps in providing adequate amount of iodine to treat and prevent iron deficiency in iron deficient subjects when 10-15 $\mathrm{g}$ of salt is consumed a day Sattrarzadeh \& Zlotkin. ${ }^{19}$ A randomized, controlled and double-blind trial to test the efficacy of double fortified salt as a mechanism to improve iron status in the age group of 19-55 yrs, $(n=217)$ among female tea pluckers, from West Bengal, India. The subjects were randomly assigned to receive iodized salt (control) or double fortified salt for a duration of ten months. Blood indicators of iron status were measured at initial and final stage, including haemoglobin, serum ferritin, and serum transferring receptor. Iodine status was also determined at initial and final stage by urinary iodine concentration. The study concluded that subjects consuming double fortified salt had significant improvement in iron status relative to control group ${ }^{20}$ conducted.

\section{Conclusion \& recommendations}

Salt is an effective food vehicle for fortification of essential micronutrients to combat the nutritional diseases like Iodine Deficiency disorders (IDD) and Iron Deficiency Anemia (IDA). Double fortified salt is enriched with potassium iodate and ferrous sulphate that helps to improve the nutritional status of vulnerable group along with lowcost benefit ratio. Moreover, double fortified salt is also stable under different storage conditions and maximum amount of nutritional content persisting to iron and iodine is observed among the plastic containers after a period of six months. Moreover, usage of double fortified salt at an end stage of cooking increasing the bioavailability of micro-nutrients. To combat IDD and IDA the provision and use of double fortified salt in Mid day meal should be made mandatory to improve the nutritional status of children and should be made one of the essential commodities in Public Distribution System to improve the health of family members. In this context, public awareness about double fortified salt should be channelized through mass media like radio, news paper, magazines, internet etc. Nutritional counselling of school and college going students regarding the importance of different nutrients in the diet and their relationship in preventing diseases should also be a part of educational curriculum. Moreover, Community health camps should also be channelized to cover at risk population.

\section{Acknowledgments}

None.

\section{Conflict of interest}

The author declares there is no conflict of interest.

\section{References}

1. World Health Organisation. Worldwide prevalence of anaemia 1993 2005. WHO global database on Anaemia. Geneva; 2008.

2. Bathla S, Grover K. Availability of iron and iodine from commonly consumed recipes using double fortified salt. Asian J Dairy Food and Research. 2016;35(3):251-54. 
3. Bathla S, Grover K. Impact of double fortified salt(DFS) supplementation on the nutritional profile: Anaemic Adolescent Girls. Chemical Science Review and Letters. 2017;(23):1630-1637.

4. Bathla S, Grover K. Effect of supplementation of double fortified salt in Mid Day Meal Programme on the nutritional status of anaemic children. Indian Journal of Agricultural Biochemistry. 2017;29(2):189-94.

5. Sivakumar B, Nair KM. Double fortified salt at crossroads. Indian $J$ Pediatr. 2002;69(7):617-623.

6. Wegmüller R, Camara F, Zimmermann MB, et al. Salt Dual-Fortified with Iodine and Micronized Ground Ferric Pyrophosphate Affects Iron Status but Not Haemoglobin in Children in Côte d'Ivoire. J Nutr. 2006;136(7):1814-1820.

7. Bathla S, Grover K, Storage stability of double fortified salt International Journal of Science and Research. 2015;4(5):1421-1424.

8. Joshi K, Nair S. Prevalence of iodine ad iron malnutrition among rural school children of Gujrat, India. Semantic Scholar. 2011;2:584-92.

9. Oshinowo T, Diosady LL, Yusufali R, et al. An Investigation of the Stability of Double Fortified Salt during Storage and Distribution in Nigeria. International Journal of Food Engineering. 2007;3(4):15563758 .

10. Mehansho H. Iron fortification technology development: New approaches. Journal of Nutrition. 2006;136:1059-1063.

11. Yusufali R, Oshinowo T, Laleye L. A study of storage and distribution of double fortified salts in Kenya. Journal of Food Engineering. 2006;76(4):547-556.

12. Romita D, Cheng Y Land, Diosady LL. Microencapsulation of Ferrous Fumarate for the Production of Salt Double Fortified with Iron and Iodine. Intern J Fd Engin. 2011;7(3):1556-3758.
13. Kumar V, Rajagopalan M, Bhagwat IP, et al. A multicenter community study on the efficacy of double-fortified salt. Fd and Nutr Bull. 2007;28(1):100-108.

14. Abizari A R, Moretti D, Zimmermann MB, et al. Whole cowpea meal fortified with NaFeEDTA reduces iron deficiency among Ghanaian school children in a malaria endemic area. J Nutr. 2012;142(10): 18361842 .

15. Nayak B, KM. In vitro bioavailability of iron from wheat flour fortified with ascorbic acid, EDTA and sodium hexametaphosphate, with or without iron. Food Chemistry. 2003;80(4):545-550.

16. Zimmermann MB, Zeder C, Chaouki N, et al. Dual fortification of salt with iodine and microencapsulated iron: a randomized, double-blind, controlled trial in Moroccan schoolchildren. American Journal of Clonical Nutrition. 2003;77(2):425-432.

17. Zimmermann MB, Winichagoon P, Gowachirapan S, et al. Comparison of the efficacy of wheat-based snacks fortified with ferrous sulfate, electrolytic iron, or hydrogen-reduced elemental iron: randomized, double-blind, controlled trial in Thai women. Am J Clin Nutr. 2005;82(6):1276-1282

18. Hurrell R. Use of ferrous fumurate to fortify foods for infants and young children. Nutr Rev. 2010;68(9):522-530.

19. Sattrarzadeh M, Zlotkin SH. Iron is well absorbed by healthy adults after ingestion of double fortified (iron and dextran coated iodine) table salt and urinary iodine excretion is unaffected. Journal of Nutrition. 1999;129(1):117-121

20. Hammons J, Venkatramanan S, Haas JD. Effect of iodine-iron interactions on iodine status in double-fortified salt intervention with female Indian tea pluckers. The Faseb J. 2012;26(1):114 